\title{
Stage III Small Intestinal, Esophageal, Colorectal, Mesenteric, and Peritoneal Gastrointestinal Stromal Tumor AJCC v8
}

National Cancer Institute

\section{Source}

National Cancer Institute. Stage III Small Intestinal, Esophageal, Colorectal, Mesenteric, and Peritoneal Gastrointestinal Stromal Tumor AJCC V8. NCI Thesaurus. Code C136783.

Stage III includes: IIIA: (T1, N0, M0, High Mitotic Rate); (T4, N0, M0, Low Mitotic Rate); IIIB: (T2, NO, MO, High Mitotic Rate); (T3, NO, MO, High Mitotic Rate); (T4, NO, MO, High Mitotic Rate). T1: Tumor measuring $2 \mathrm{~cm}$ or less. T2: Tumor measuring more than $2 \mathrm{~cm}$ but not more than $5 \mathrm{~cm}$. T3: T umor measuring more than $5 \mathrm{~cm}$ but not more than 10 $\mathrm{cm}$. T4: Tumor measuring more than $10 \mathrm{~cm}$ in greatest dimension. N0: No regional lymph node metastasis or unknown lymph node status. M0: No distant metastasis. High Mitotic Rate: Over 5 mitoses per 5 square millimeters, or per 50 HPF. Low Mitotic Rate: Five or fewer mitoses per 5 square millimeters, or per 50 HPF. (AJCC 8th ed.) 